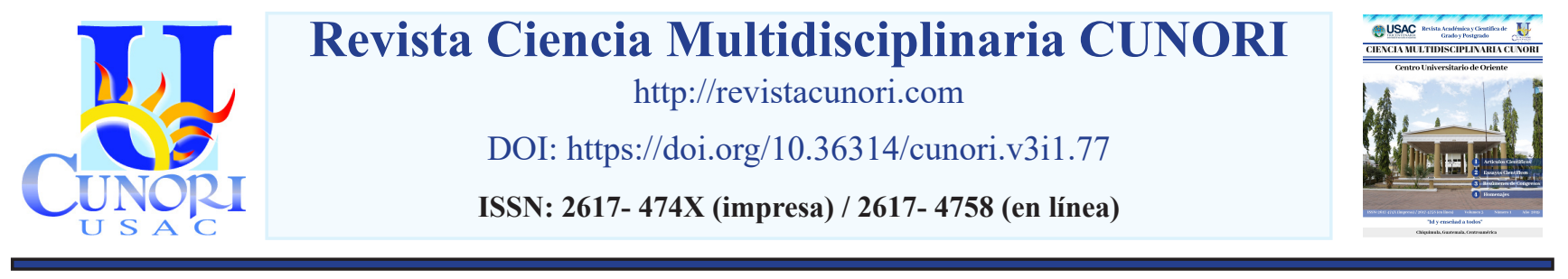

Como citar el artículo

Salguero, L., Mazariegos, E., Romero, J., \& Pineda, R. (2019). Caracterización clínica de diagnósticos de pacientes pediátricos con dengue. Revista Ciencia Multidisciplinaria CUNORI 3(1). 29-41. DOI: https://doi.org/10.36314/cunori.v3i1.77

\title{
Caracterización clínica de diagnósticos de pacientes pediátricos con dengue
}

\section{Clinical characterization of diagnoses of pediatric patients with dengue}

\author{
Luisana Salguero*, Edvin Mazariegos, Johanna Romero, Roberto Pineda \\ Centro Universitario de Oriente (CUNORI), Universidad de San Carlos de Guatemala \\ Recibido: 21 de julio de 2018 / Revisión: 20 de agosto de 2018 / Aceptado: 10 de enero de 2019 \\ Disponible en internet el 30 de agosto de 2019 \\ *Autor para correspondencia. \\ Correo electrónico: lui_0993@outlook.com
}

Resumen

E 1 dengue es una enfermedad viral, que se transmite por mosquitos hembras principalmente de la especie Aedes aegypti. Puede presentarse de manera asintomática o con un espectro clínico amplio que incluye la clasificación de: dengue sin signos de alarma, dengue con signos de alarma y dengue grave. La detección oportuna y el acceso a la asistencia médica adecuada disminuyen las tasas de mortalidad por debajo del 1\%. En 2015, se notificaron 2.35 millones de casos tan solo en la Región de las Américas. Se realizó un estudio descriptivo retrospectivo de 205 pacientes sobre la caracterización clínica y de métodos diagnósticos de pacientes pediátricos con dengue y dengue grave, ingresados en el Hospital Regional de Zacapa en los años 2013 a 2017 . De 205 pacientes pediátricos ingresados con diagnóstico de dengue, $46 \%$ corresponde a dengue con signos de alarma, 30\% sin signos de alarma y $24 \%$ a dengue grave. El signo más frecuente en pacientes con dengue con signos y sin signos de alarma fue fiebre $100 \%$, y vómitos con $24 \%$, el síntoma predominante fue el dolor abdominal con $48 \%$ en pacientes con dengue grave y con signos y sin signos de alarma, el hallazgo ultrasonográfico más frecuente en dengue con signos y sin signos de alarma fue el engrosamiento de la pared de la vesícula biliar $87 \%$ y en dengue grave fue hepatomegalia con 31\%, el 100\% presentó recuento bajo de plaquetas.

Palabras clave: dengue con signos de alarma, dengue sin signos de alarma, dengue grave

Abstract

$\mathrm{D}$ engue is a viral disease that is transmitted by female mosquitoes, mainly of the Aedes aegypti species. It can occur asymptomatically or with a broad clinical spectrum that includes the classification of: dengue without warning signs, dengue with warning signs and severe dengue. Timely detection and access to adequate medical care decrease mortality rates below $1 \%$. In 2015, 2.35 million cases were reported only in the Region of the Americas. A retrospective descriptive study was conducted of 205 patients on the clinical characterization and diagnostic methods of pediatric patients with dengue and severe dengue, admitted to the Zacapa Regional Hospital in the years 2013 to 2017. Of 205 pediatric patients admitted with a diagnosis of dengue, $46 \%$ corresponds to dengue with warning signs, $30 \%$ without warning signs and $24 \%$ to severe dengue. The most frequent sign in patients with dengue with signs and without warning signs was $100 \%$ fever, and vomiting with $24 \%$, the predominant symptom was abdominal pain with $48 \%$ in patients with severe dengue and with signs and without warning signs, the most frequent ultrasonographic finding in dengue with signs and without warning signs was the thickening of the gallbladder wall $87 \%$ and severe dengue was hepatomegaly with $31 \%, 100 \%$ had low platelet count.

Keywords: dengue with warning signs, dengue without warning signs, severe dengue 


\section{Introducción}

El dengue es una virosis pandemiógena, que se está propagando rápidamente en muchas regiones del mundo. Se transmite por mosquitos hembras principalmente de la especie Aedes aegypti, y en menor grado, de Aedes albopictus (OMS 2017). Hasta el momento se han descrito cuatro serotipos de este virus que circulan principalmente en países del sudeste asiático, del Pacifico occidental y de América Latina y el Caribe, por lo que la enfermedad se considera tropical (Kouri 2006). La infección puede cursar en forma asintomática o expresarse con un espectro clínico amplio que incluye la clasificación de: dengue sin signos de alarma, dengue con signos de alarma y dengue grave. Dentro de los signos de alarma se encuentra: dolor abdominal, hemorragia en mucosas, trombocitopenia, letargia, hipoalbuminemia y hematocrito elevado. Los criterios para dengue grave son: extravasación severa de plasma, expresada en choque hipovolémico, o por dificultad respiratoria debido al exceso de líquido acumulado en el pulmón; Hemorragias severas y afectación de órganos (OPS, 2016).

En 1954, se describió el dengue grave (conocido anteriormente como dengue hemorrágico) durante una epidemia de la enfermedad en Filipinas y Tailandia. Hoy en día, afecta a la mayor parte de los países de Asia y América Latina y se ha convertido en una de las causas principales de hospitalización y muerte en los niños y adultos de dichas regiones (Méndez y González, 2006). La mitad de la población del mundo corre el riesgo de contraer la enfermedad. En 2015, se notificaron 2.35 millones de casos tan solo en la Región de las Américas, de los cuales más de 10,200 casos fueron diagnosticados como dengue grave y provocaron 1,181 defunciones (OMS, 2017).

El estudio tiene como objetivo determinar las características del paciente pediátrico con dengue sin signos de alarma, dengue con signos de alarma y dengues graves e ingresados en el hospital regional de Zacapa en el periodo de 2013 a 2017.

\section{Materiales y métodos}

Se realizó un estudio descriptivo retrospectivo sobre la caracterización clínica y de métodos diagnósticos de pacientes pediátricos con dengue y dengue grave, ingresados en el Hospital Regional de Zacapa en el periodo de 2013 a 2017. Se incluyen 205 expedientes clínicos de pacientes con diagnóstico de dengue y dengue grave de 0 a 13 años, en el periodo establecido, se excluyeron expedientes incompletos o en mal estado, pacientes mayores de 13 años, pacientes con pruebas negativas para dengue y sin exámenes complementarios.

Las variables estudiadas fueron Dengue (sin signos de alarma y con signos de alarma) Dengue grave, caracterización clínica (días con fiebre, petequias, hemorragias, hipotensión, prueba de torniquete, dolor abdominal y dificultad respiratoria) y métodos diagnósticos (pruebas de laboratorio y hallazgos ultrasonográficos).

Para la recolección de datos se revisaron los expedientes clínicos de todos los pacientes que cumplen los criterios de inclusión durante el período establecido, esta información se registró en una boleta de recolección de datos por número de registro hospitalario de cada paciente, los datos recolectados fueron tabulados y expresados gráficamente. 


\section{Resultados}

Edad de los pacientes pediátricos con diagnóstico de dengue sin signos de alarma, dengue con signos de alarma y dengue grave

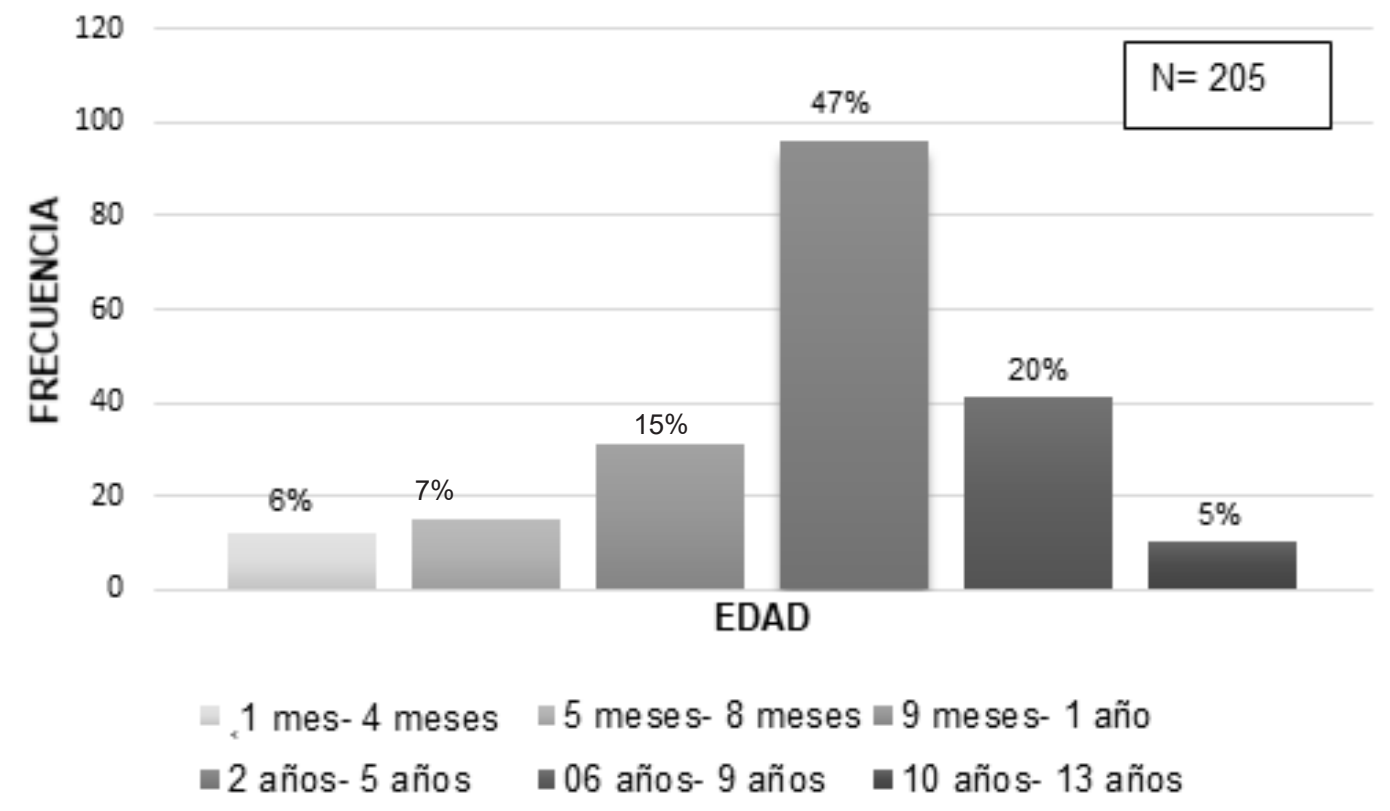

Figura 1: Distribución de pacientes pediátricos con diagnóstico de dengue y dengue grave ingresados en el Hospital Regional de Zacapa, según la edad, en el período del año 2013 al año 2017.

La edad que se observa con mayor frecuencia en los pacientes pediátricos con diagnóstico de dengue y dengue grave es la comprendida entre 2- 5 años representando el 47\% (96), seguido de 6-9 años con $20 \%(41)$. 
El sexo de los pacientes pediátricos con dengue sin signos de alarma y con signos de alarma y dengue grave

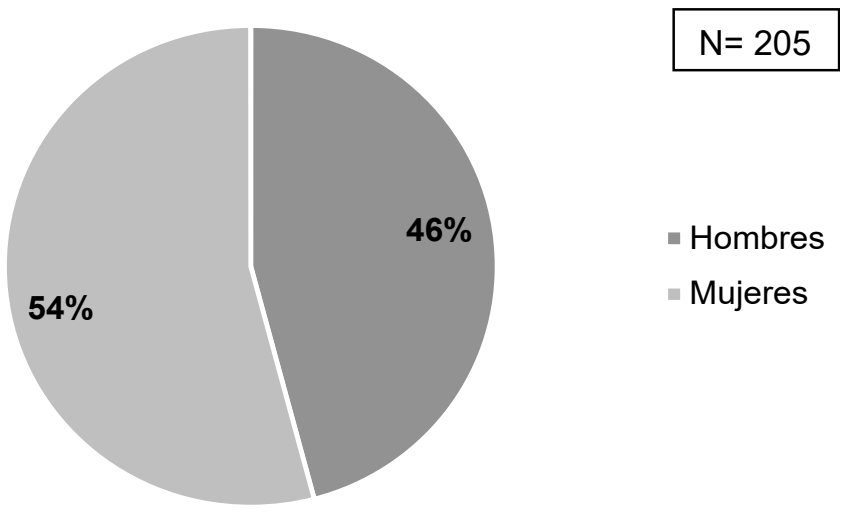

Figura 2: Distribución de los pacientes pediátricos con diagnóstico de dengue y dengue grave ingresados en el Hospital Regional de Zacapa, según el género, en el período del año 2013 al año 2017.

De 205 pacientes pediátricos con dengue y dengue grave, 54\% corresponden al sexo femenino (111), y un $46 \%$ al sexo masculino (94).

Procedencia por departamento de los pacientes pediátricos con dengue sin signos de alarma y con signos de alarma y dengue grave

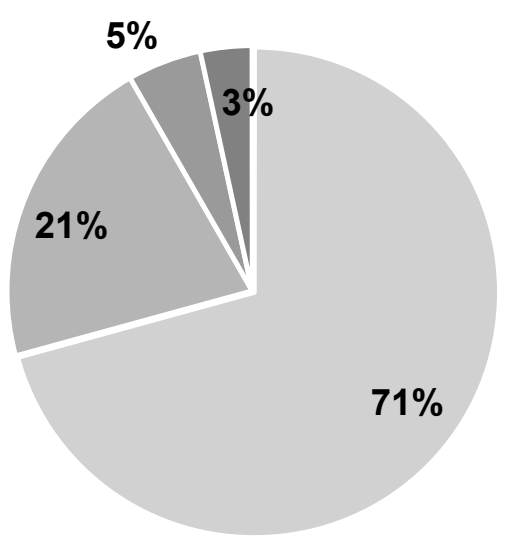

$$
\begin{aligned}
& \text { N=205 } \\
& \text { - Zacapa } \\
& \text { - El Progreso } \\
& \text { - Chiquimula } \\
& \text { - Izabal }
\end{aligned}
$$

Figura 3: Distribución de los pacientes pediátricos con diagnóstico de dengue y dengue grave ingresados en el Hospital Regional de Zacapa, según la procedencia por departamento, en el período del año 2013 al año 2017.

El departamento con mayor ingreso hospitalario de pacientes pediátricos con diagnóstico de dengue y dengue grave es Zacapa representando un 71\% (145), seguido del departamento El Progreso con un $21 \%$ (43) y en menor frecuencia se encuentra el departamento de Izabal con un 3\% (7). 
Procedencia por municipio de los pacientes pediátricos con dengue sin signos de alarma y con signos de alarma y dengue grave

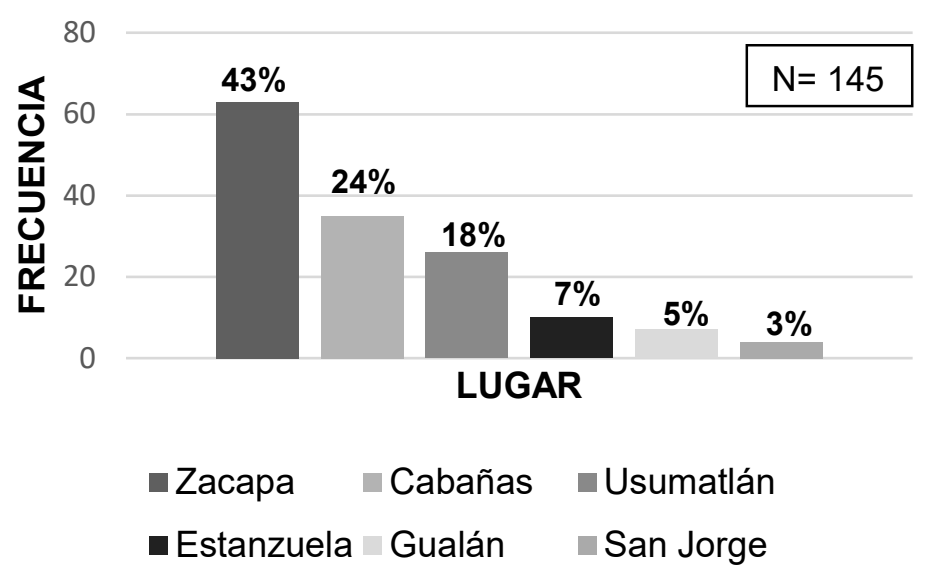

Figura 4: Distribución de los pacientes pediátricos con diagnóstico de dengue y dengue grave ingresados en el Hospital Regional de Zacapa, según la procedencia por municipio en el período del año 2013 al año 2017.

Dentro de los municipios con mayor ingreso hospitalario se encuentra la cabecera departamental de Zacapa representada con 43\% (63), seguido del municipio de Cabañas con 24\% (35) y en menor frecuencia se encuentra el municipio de San Jorge con 3\% (4).

\section{Clasificación de dengue}

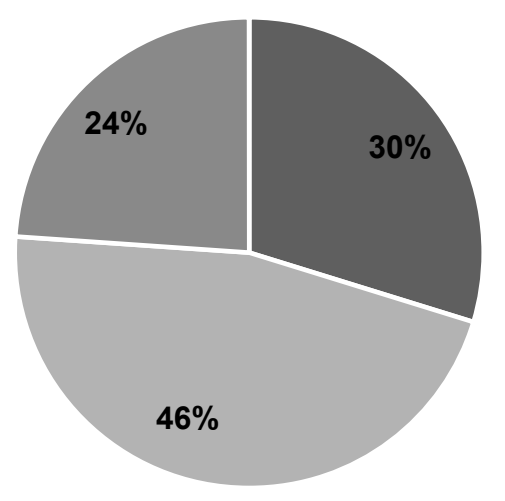

$\mathrm{N}=205$

- Dengue sin signos de alarma

- Dengue con signos de alarma

- Dengue grave

Figura 5: Distribución de pacientes pediátricos con diagnóstico de dengue en el Hospital Regional de Zacapa, según la clasificación, en el período del año 2013 al año 2017.

De 205 pacientes pediátricos ingresados en el Hospital Regional de Zacapa con diagnóstico de dengue, $46 \%$ corresponde a la clasificación de dengue con signos de alarma (95), seguido por 30\% de dengue sin signos de alarma (61) de los cuales presentaban comorbilidades. 


\section{Características clínicas}
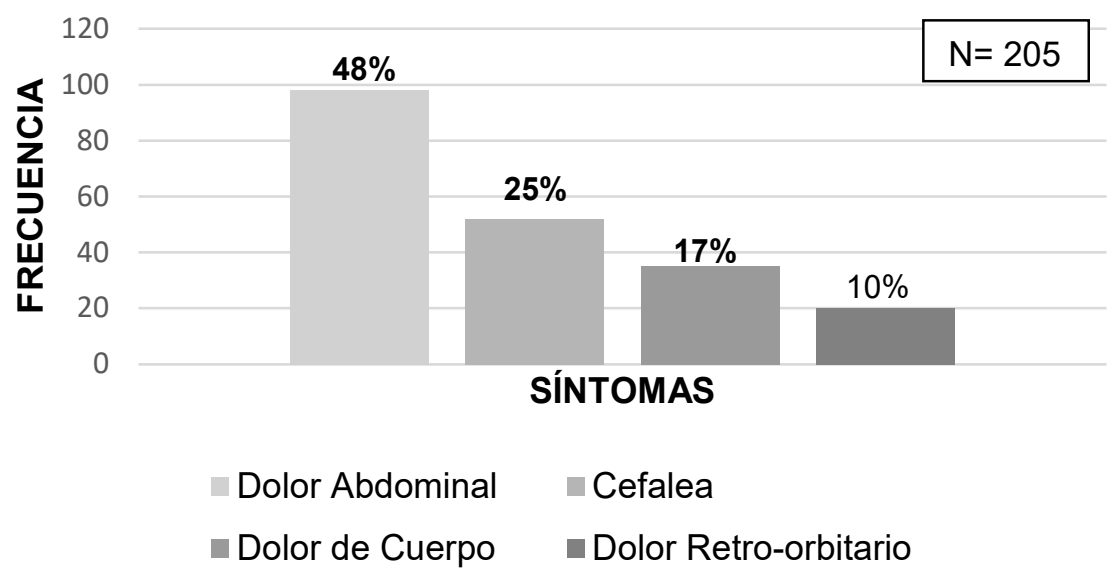

Figura 6: Distribución de pacientes pediátricos con diagnóstico de dengue y dengue grave ingresados en el Hospital Regional de Zacapa, según los síntomas, en el período del año 2013 al año 2017.

El síntoma con mayor frecuencia representado en los pacientes pediátricos con dengue grave y dengue con signos y sin signos de alarma es el dolor abdominal con $48 \%$ (98), seguido por cefalea con $25 \%$ (52) y el síntoma menos frecuente fue el dolor retro-orbitario con $10 \%(20)$.

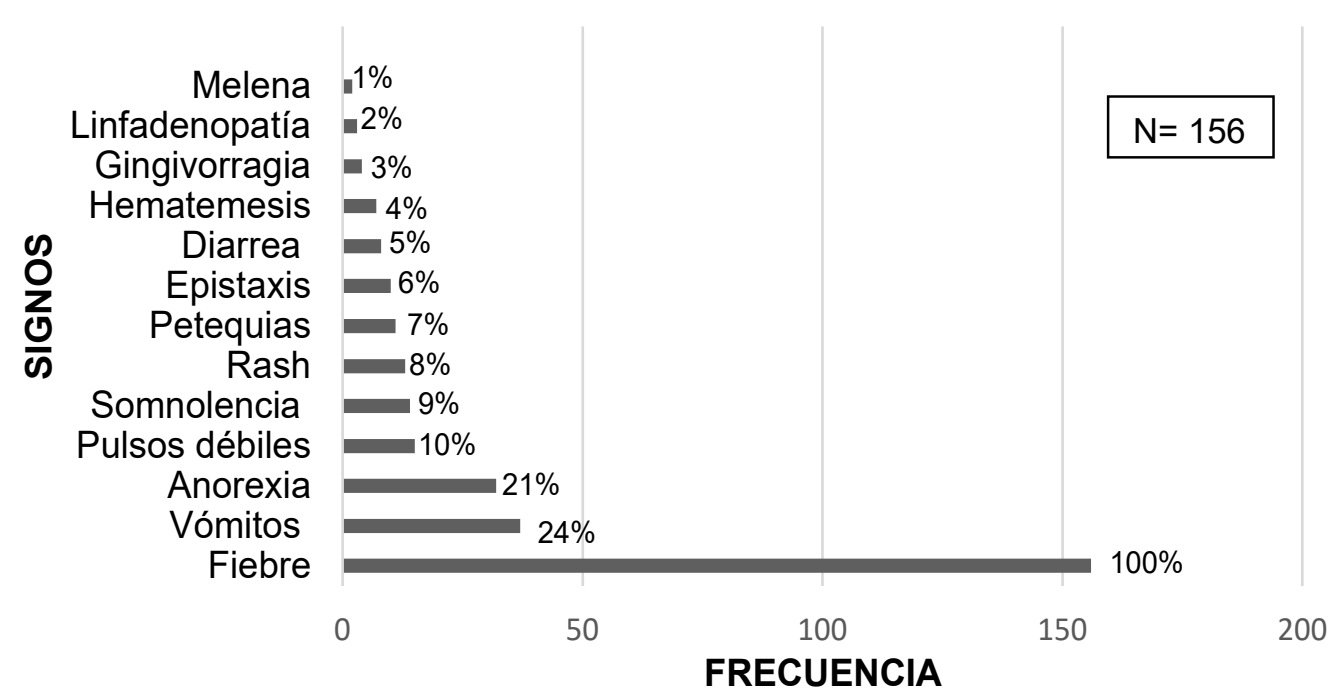

Figura 7: Distribución de pacientes pediátricos con diagnóstico de dengue con signos y sin signos de alarma ingresados en el Hospital Regional de Zacapa, según los signos predominantes, en el período del año 2013 al año 2017.

Los signos más frecuentes en los pacientes con dengue sin signos y con signos de alarma, la fiebre predomina con 100\% (156), seguido por los vómitos con 24\% (37), la anorexia se ve representada con $21 \%$ (32 casos). 


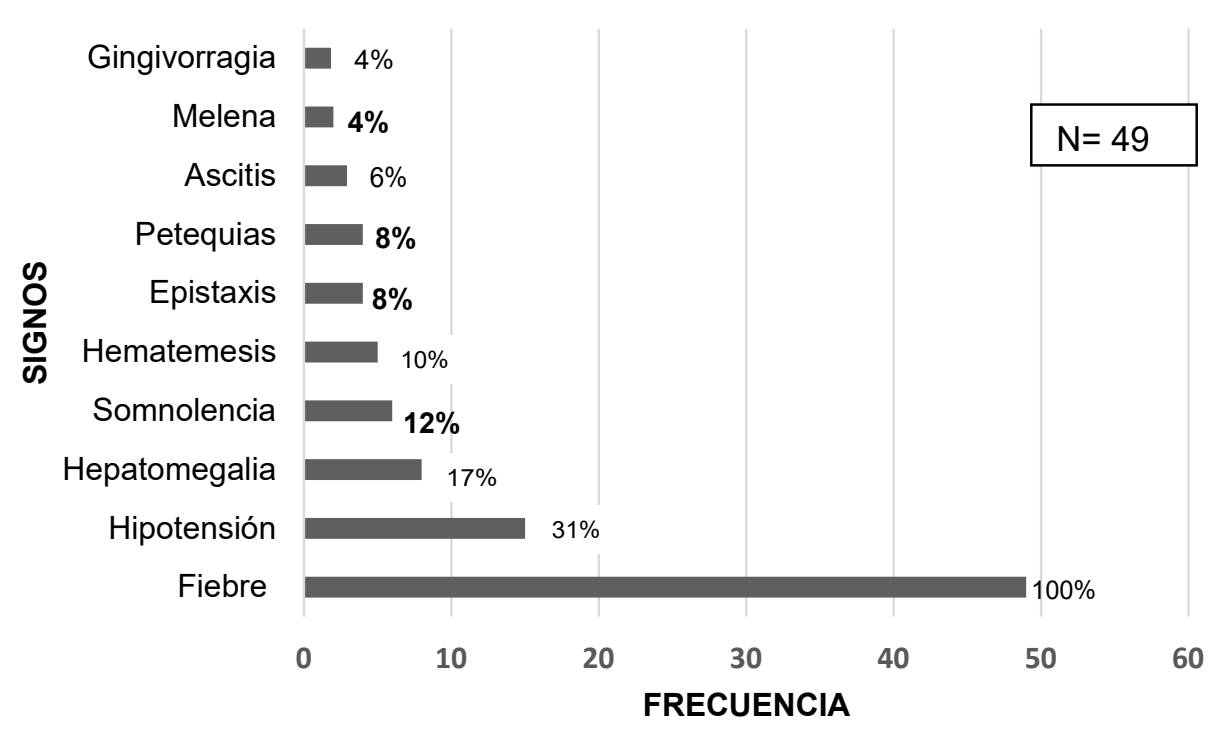

Figura 8: Distribución de pacientes pediátricos con diagnóstico de dengue grave ingresados en el Hospital Regional de Zacapa, según signos predominantes, en el período del año 2013 al año 2017.

El signo más frecuente en los pacientes pediátricos con dengue grave fue fiebre con 100\% (49 casos), seguido de hipotensión con 31\% (15), hepatomegalia se encontró con 17\% (8).

\section{Métodos diagnósticos}

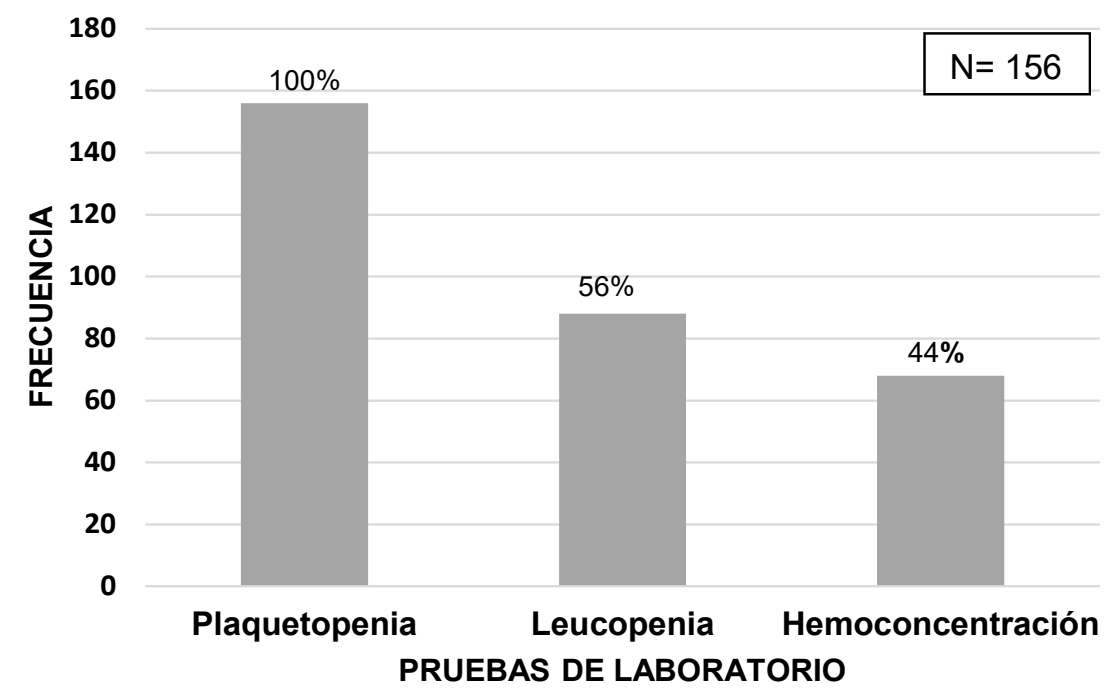

Figura 9: Distribución de pacientes pediátricos con diagnóstico de dengue con signos y sin signos de alarma ingresados en el Hospital Regional de Zacapa, según las pruebas de laboratorio realizadas, en el período del año 2013 al año 2017.

Dentro de las pruebas de laboratorio realizadas a los pacientes pediátricos, la plaquetopenia es la que se presenta con mayor frecuencia con $100 \%$ (156), seguido por leucopenia con $56 \%$ (88) y en menor frecuencia hemoconcentración con 44\% (68). 


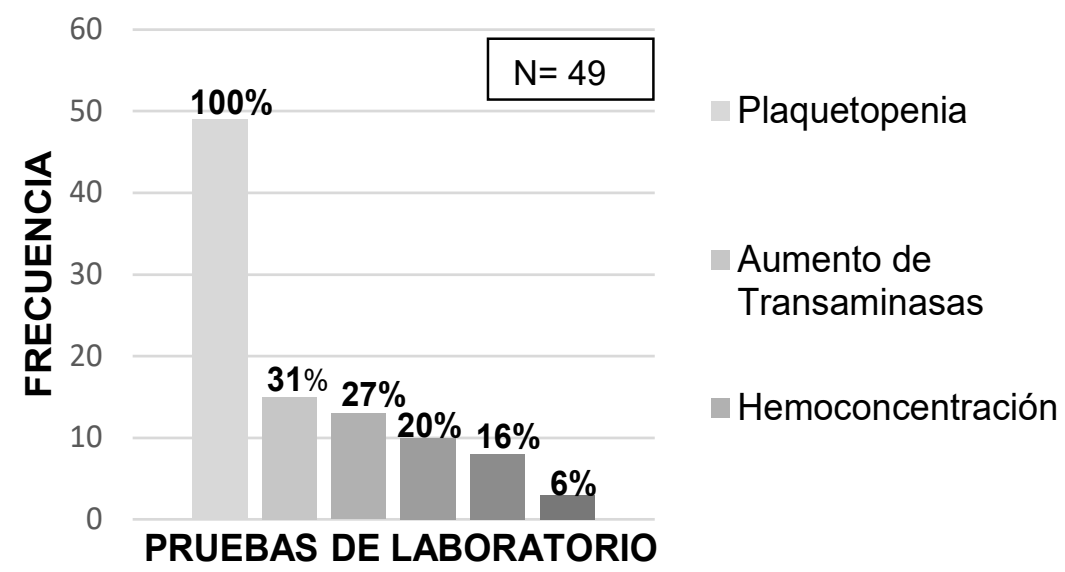

Figura 10: Distribución de pacientes pediátricos con diagnóstico de dengue grave ingresados en el Hospital Regional de Zacapa, según las pruebas de laboratorio realizadas, en el período del año 2013 al año 2017.

La prueba de laboratorio que mayor alteración presenta en los pacientes pediátricos con dengue grave es plaquetopenia representando $100 \%$ (49), seguido por aumento de transaminasas con $31 \%$ (15).

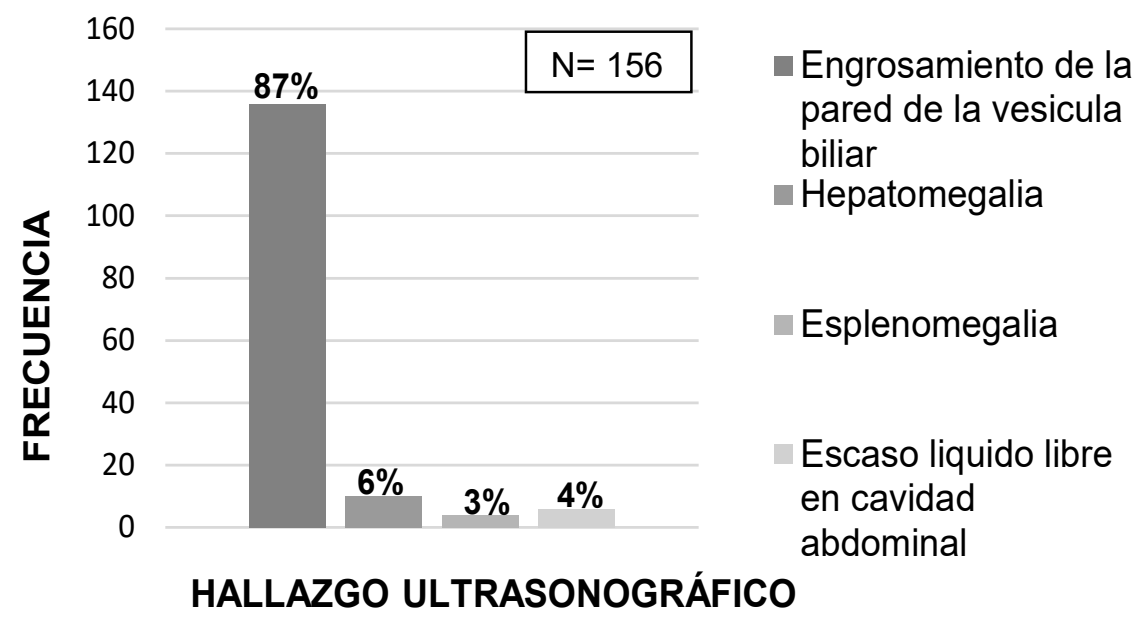

Figura 11: Distribución de pacientes pediátricos con diagnóstico de dengue con signos y sin signos de alarma ingresados en el Hospital Regional de Zacapa, según hallazgos ultrasonográficos, en el período del año 2013 al año 2017.

Con respecto a los hallazgos ultrasonográficos, el engrosamiento de la pared de la vesícula biliar es la que se presenta con mayor frecuencia con 87\% (136) en los pacientes pediátricos con dengue sin signos y con signos de alarma, seguido por hepatomegalia con $6 \%(10)$. 


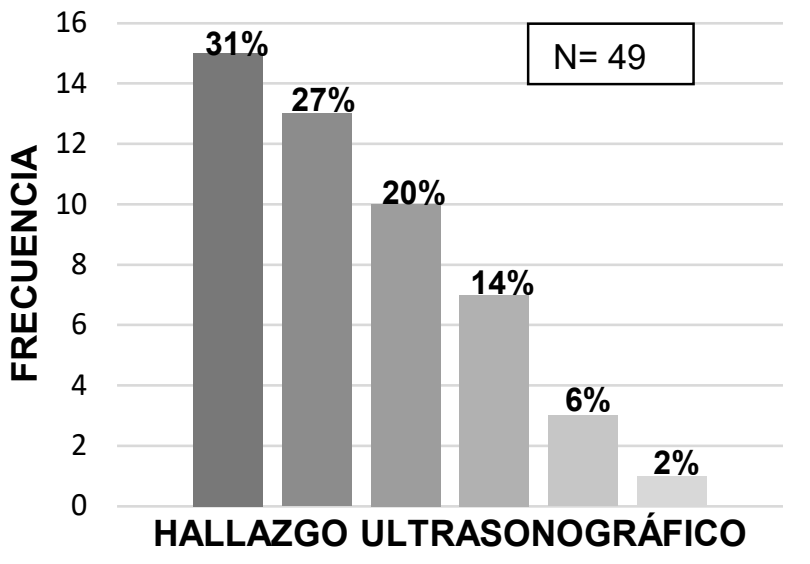

- Hepatomegalia

- Derrame pleural

Engrosamiento de la pared de la vesicula biliar

$\square$ Abundante liquido libre en cavidad abdominal

Figura 12: Distribución de pacientes pediátricos con diagnóstico de dengue grave ingresados en el Hospital Regional de Zacapa, según hallazgos ultrasonográficos, en el período del año 2013 al año 2017.

Dentro de los principales hallazgos ultrasonográficos en dengue grave predominó hepatomegalia con $31 \%$ (15), seguido por derrame pleural con 27\% (13), el hallazgo menos frecuente fue liquido libre en la cavidad pélvica con $2 \%$ (1).

\section{Condición de egreso}

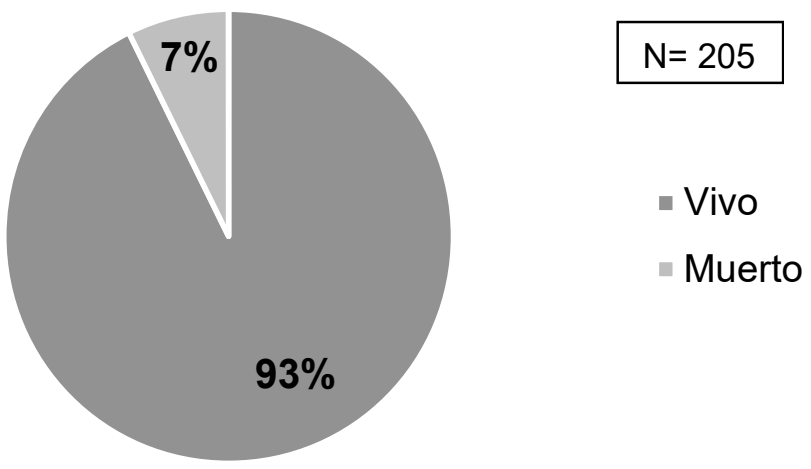

Figura 13: Distribución de pacientes pediátricos con diagnóstico de dengue y dengue grave ingresados en el Hospital Regional de Zacapa, según condición de egreso, en el período del año 2013 al año 2017.

La principal condición de egreso fue vivo con 93\% (190), y el 7\% restante corresponde a la condición de egreso de los pacientes fallecidos los cuales tenían como diagnóstico establecido dengue grave (15). Con una tasa de letalidad de $7 \%$. 


\section{Causa de muerte}

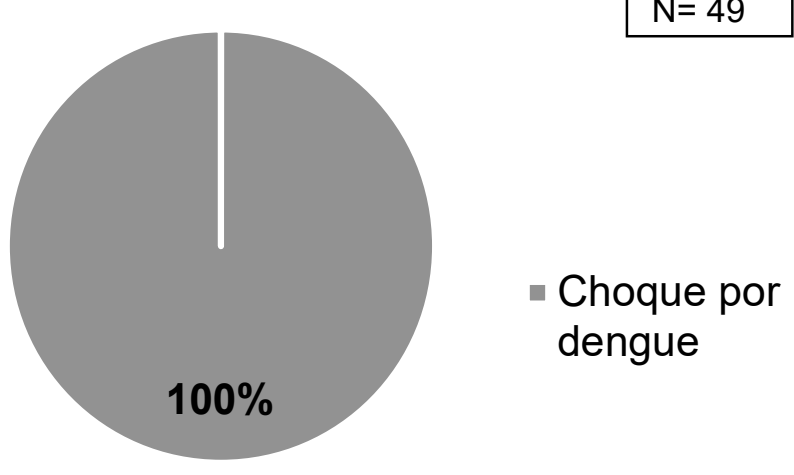

Figura 14: Distribución de pacientes pediátricos con diagnóstico dengue grave ingresados en el Hospital Regional de Zacapa, según causa de muerte, en el período del año 2013 al año 2017.

La única causa de muerte de los 15 pacientes fallecidos con diagnóstico de dengue grave fue choque por dengue lo que representa el $100 \%$.

\section{Discusión}

Se realizó un estudio de 205 pacientes pediátricos con dengue y dengue grave atendidos en el Hospital Regional de Zacapa en el periodo del año 2013 al año 2017, en el cual se puede observar que el rango de edad más frecuente en esta patología es el de 2 a 5 años. Con respecto al sexo el femenino predomina con 54\%, de los casos, según (González \& Méndez, 2002), el sexo tiene una ligera predisposición como factor de riesgo.

En Colombia también se realizó un estudio por (Matta et al., 2016), en el cual sus resultados muestran que el sexo femenino se presenta con mayor frecuencia en los pacientes con diagnóstico de dengue, coincidiendo con el presente estudio. En este estudio se observó que el mayor ingreso hospitalario es de los pacientes pediátricos con diagnóstico de dengue con signos de alarma con $46 \%$, esto se debe a que estos pacientes deben permanecer bajo vigilancia hospitalaria por lo menos por 72 horas (Pizarro, 2009).

Según el (MSPAS, 2017), el departamento de Zacapa e Izabal se encuentra entre los departamentos con alta incidencia de casos de dengue. Además, debido al clima tropical que este departamento posee lo hace susceptible de padecer esta patología. En este estudio se observa que según la procedencia de los pacientes pediátricos con diagnóstico de dengue y dengue grave la mayoría son del departamento de Zacapa con $71 \%$ y con menor frecuencia se observa Izabal con 3\%.

En cuanto a la procedencia por municipios la mayor población de pacientes proviene de la cabecera departamental Zacapa con $43 \%$ de los casos, esto es debido a la cercanía al centro asistencial, el segundo municipio con más ingresos hospitalarios es Cabañas con $24 \%$. 
En cuanto a los síntomas más frecuentes de dengue y dengue grave, el dolor abdominal predominó con $48 \%$, seguido por cefalea con $25 \%$. Esto se debe a que el dolor abdominal es una de las manifestaciones más importantes en el dengue con signos de alarma y dengue grave, debido a la extravasación de plasma hacia el tercer espacio lo cual puede provocar ascitis. Un estudio realizado en el Hospital General San Juan de Dios y Roosevelt, Guatemala; concuerda con esta investigación debido a que el síntoma más frecuente fue también dolor abdominal con 74\%, según (Pérez, et al., 2012).

El dengue es una virosis que puede cursar de forma asintomática o manifestarse con un espectro febril inespecífico. (González \& Méndez, 2002). El signo más frecuente dentro de la clasificación de dengue con signos y sin signos de alarma fue fiebre con $100 \%$, seguido por los vómitos con $24 \%$. Y en la población con dengue grave también se observó que el signo predominante fue fiebre con (100\%), seguido de hipotensión con 31\%. (Matta et al., 2016), en su estudio realizado en Colombia demuestran también que la fiebre predominó como signo en pacientes con dengue en 100\%.

Respecto a las pruebas de laboratorio, la plaquetopenia ocupa especial importancia debido a que predominó en 100\%, dentro de las 3 clasificaciones de dengue. Según la (OPS, 2016), esto suele preceder a la extravasación de plasma. Los pacientes con dengue con signos y sin signos de alarma presentaron en menor frecuencia una hemoconcentración con $44 \%$. En dengue grave se observó que la segunda prueba de laboratorio más frecuente es el aumento de transaminasas con $31 \%$, esto se debe al daño a órgano blanco que produce esta patología, este es uno de los principales criterios para poder clasificar a un paciente con dengue grave. Este estudio demuestra que dentro de los hallazgos ultrasonográficos más frecuentes el engrosamiento de la pared de la vesícula biliar predomina con $87 \%$, seguido por hepatomegalia con $6 \%$.

Durán et al. (2010), indican que el bazo presenta aumentó de tamaño por congestión y su consistencia es blanda y todos los órganos tienen algún grado de edema e hiperemia. Dentro de los principales hallazgos ultrasonográficos en dengue grave predominó hepatomegalia con $31 \%$, seguido por derrame pleural con $27 \%$, el hallazgo menos frecuente fue líquido libre en la cavidad pélvica con $2 \%$. Esto sucede por la fuga plasmática y el daño a órganos blancos.

(Núñez, 2013), indica que, en su estudio realizado en Ecuador, el edema perivesicular y el derrame pleural son los hallazgos ultrasonográficos predominantes. En contraste con el presente estudio en donde predomina el engrosamiento a la pared de la vesícula biliar y hepatomegalia como los principales hallazgos ultrasonográficos.

Los signos clínicos de dengue más trombocitopenia y hemoconcentración, manifestada por hematocrito en ascenso, son suficiente para sospechar de diagnóstico clínico de dengue grave (González \& Méndez, 2002). En este estudio $78 \%$ presentó elevación del hematocrito a las 48 horas de su ingreso hospitalario, $16 \%$, permaneció con el valor del hematocrito normal según los rangos para su edad. Según los valores de referencia del manual Harriet Lane de pediatría. Con respecto a la condición de egreso, $93 \%$ egreso mejorado y sin ninguna anomalía y el otro $7 \%$ que tenía como diagnostico establecido dengue grave falleció y su principal causa de muerte fue choque por dengue, con una tasa de letalidad del $7 \%$. 


\section{Agradecimientos}

Este trabajo ha sido posible gracias al apoyo y asesoría de las autoridades de la carrera de médico y cirujano del Centro Universitario de Oriente y al apoyo del Hospital Regional de Zacapa.

\section{Referencias bibliográficas}

Durán, C., Lanza, T. \& Plata, J. (2010). Fisiopatología y diagnóstico del dengue. Revista Médica Hondureña 78 (3): 136-141. Recuperado de http://cidbimena.desastres.hn/RMH/pdf/2010/pdf/Vol78-32010-8.pdf

González, G. \& Méndez, B. (2002). Dengue. Curso Continuo de Actualización en Pediatría 2 (mod.1): 5-20. Recuperado de https://scp.com.co/precop-old/precop_files/modulo_2_vin_1/precop_ano2_ mod1_dengue.pdf

Kouri, G. (2006). El dengue, un problema creciente de salud en las Américas. Revista Panamericana de la Salud 19 (3): 143-145. https://doi.org/10.1590/S1020-49892006000300001

Matta, L., Barbosa, M. \& Morales, C. (2016). Caracterización clínica de pacientes que consultaron por dengue en un hospital de tercer nivel en Cali, Colombia, 2013. Revista Biomédica 36 (1): 133-139. https://doi.org/10.7705/biomedica.v36i1.2627

Méndez, A. \& González, G. (2006). Manifestaciones clínicas inusuales del dengue hemorrágico en niños. Revista Biomédica 26: 61-70. . https://doi.org/10.7705/biomedica.v26i1.1395

Ministerio de Salud Pública y Asistencia Social -MSPAS. (2017). Semana epidemiológica 11: del 13 al 19 de marzo de 2017. Guatemala, departamento de Epidemiologia. p.7 Recuperado de http://epidemiologia.mspas.gob.gt/files/Publicaciones\%202017/SEMEPI\%202017/SEMEPI_11_2017.pdf

Núñez, K. (2013). Categorización de los signos de alarma en pacientes con dengue del hospital de infectología "Dr. José Daniel Rodríguez" en el periodo de enero a marzo de 2013. (Tesis de Licenciatura) Santiago de Guayaquil, Ecuador, Universidad Católica. p.10. Recuperado de http://repositorio.ucsg. edu.ec/ bitstream/3317/2085/1/T-UCSG-PRE-MED-176.pdf

Organización Mundial de la Salud -OMS. (2017). Dengue. Suiza. Recuperado de http://www.who.int/ topics/dengue/es/

Organización Panamericana de la Salud -OPS. (2016). Dengue: guías para la atención de enfermos en la región de las Américas. 2 ed. Washington. 144 p. Recuperado de http://www.hirrc.org/Gu\%C3\% ADa\%20dengue\%20OPS\%202016.pdf

Pérez, F., Monterroso, A. \& Zamora, B. (2012). Caracterización clínica y epidemiológica de dengue. (Tesis de Licenciatura). Universidad de San Carlos de Guatemala, Facultad de Ciencias Médicas, Guatemala. p.128. Recuperado de http://biblioteca.usac.edu.gt/tesis/05/05_8949.pdf

Pizarro, D. (2009). Dengue, dengue hemorrágico. Acta Pediátrica Costarricense 21 (1): 8-17. Recuperado de http://www.scielo.sa.cr/ pdf/apc/v21n1/a02v21n1 


\section{Sobre la autora}

\section{Luisana Valeria Salguero González}

Médico y Cirujano, egresada del Centro Universitario de Oriente CUNORI de la Universidad de San Carlos de Guatemala en el año 2018. Realizó la investigación que lleva como título "Caracterización clínica de diagnósticos de pacientes pediátricos con dengue".

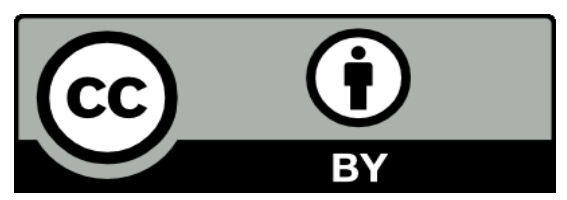

Este texto está protegido por una licencia CreativeCommons 4.0.

Usted es libre para compartir, copiar y redistribuir el material en cualquier medio o formato y adaptar el documento, remezclar, transformar y crear a partir del material para cualquier propósito, incluso comercialmente, siempre que cumpla la condición de atribución: usted debe reconocer el crédito de una obra de manera adecuada, proporcionar un enlace a la licencia, e indicar si se han realizado cambios. Puede hacerlo en cualquier forma razonable, pero no de forma tal que sugiera que tiene el apoyo del licenciante o lo recibe por el uso que hace. 